\title{
The Influence of Cognitive Biases in Production Logistics
}

\author{
Florian Knapp (D), Melanie Kessler $\mathbb{D}$, and Julia C. Arlinghaus
}

\begin{abstract}
Digitalization and the transformation of industry into Industry 4.0 is changing the character of production logistics substantially. New Logistics 4.0 technologies are largely enabling automated decision-making by machines. Human decisions are nevertheless still required. Research shows, however, that human decisions are often more biased and less rational than most logistics models assume. Decision makers and decision support system designers therefore need to understand the influence of the so-called cognitive biases on the human decisionmaking process. We contribute to the scholarship on this issue by combining the literature streams of logistics and cognitive biases. We demonstrate the influence of cognitive biases on human decision-making based on typical decisions in logistics and derive initial hypotheses.
\end{abstract}

\section{Introduction}

Industry 4.0 or the Fourth Industrial Revolution is changing all areas of manufacturing. New technologies, such as online cyber-physical systems, the Internet of Things (IoT), and cloud-based solutions, are transforming conventional man-

Julia C. Arlinghaus was affiliated to LogDynamics until 2017 as Professor of Optimization of Production and Logistics Networks at the Jacobs University Bremen, Germany

\author{
F. Knapp \\ Otto von Guericke University, Magdeburg, Germany \\ e-mail: florian.knapp@ovgu.de \\ M. Kessler \\ RWTH Aachen University, Aachen, Germany \\ e-mail: melanie.kessler@rwth-aachen.de \\ J. C. Arlinghaus ( $\square)$ \\ Otto von Guericke University, Magdeburg, Germany \\ e-mail: julia.arlinghaus@ovgu.de
}


ufacturing facilities into the so-called smart factories (Skapinyecz et al. 2018). These technologies are enabling industry to transition to full digitalization and smart manufacturing processes (Erol et al. 2016; Brettel et al. 2014; De Felice and Petrillo 2012). As digitalization increases, the field of logistics is also undergoing a major transformation (Rushton et al. 2006). Logistics is a broad field comprising the integrated planning, implementation, and control of the flow and storage of materials and products, services, information, energy, and other resources. This means that every flow into, through, and out of an organization is covered from its point of origin to its point of consumption with the aim of meeting customer demands (Johnson et al. 1999). The transformation of traditional logistics in conjunction with Industry 4.0 generally referred to as Logistics 4.0 offers logisticians new opportunities to boost efficiency and cut costs and creates new opportunities through the aforementioned digital innovations (Bamberger et al. 2017).

Figure 1 summarizes the new domains based on Logistics 4.0 and the new opportunities these new technologies provide. No longer confined to one company, industry or country, material, information and services will increasingly become a global system, as the lower half of Fig. 1 indicates. The range of activities constitutes a challenge for all businesses, though.

Logistics in general chiefly focuses on the optimization of business activities, i.e., improving the flow and storage of inventory, goods, and services through the supply chain (CSCMP 2021). Improving logistics performance in relation to the aforementioned parameters requires consideration of multiple factors.

The new technologies presented in Fig. 1 are intended to enable these logistical parameters' complex connections and assist humans with their decision-making in various logistics operations. Whereas logistics research has substantial experience with these standard rational factors, it frequently overlooks human factors. Judgment and decision-making are crucial and fundamental to the field of logistics, but humans tend to make systematic errors when making decisions, especially when they are encumbered by time pressure and uncertainty (Bazerman et al. 2002; Stanovich and West 1998). Cognitive biases are the root cause of this (Tversky and Kahneman 1974a). The phenomenon of lead time syndrome (LTS), for instance, illustrates how biased human decision-making diminishes logistics performance. Bendul and Knollmann (2016) identified several cognitive biases that influence production planners in the event they are confronted by unforeseen events, such as declining due date reliability. Planners consequently tend to adjust system parameters, such as planned lead times, in order to improve the logistics performance, albeit this actually worsens due date reliability.

This example demonstrates the effect of cognitive biases on logistics performance and illustrates the strong interrelationship between logistics and manufacturing operations. We therefore focus on production logistics in this book chapter with the intention of answering the following research question: Which types of decisions in production logistics are influenced by cognitive biases and how do these biases affect production logistics performance?

To this end, we combine the literature streams on production logistics and cognitive biases. Considering practical examples, we map the cognitive biases identified beforehand to decision situations typical to production logistics and 
Category
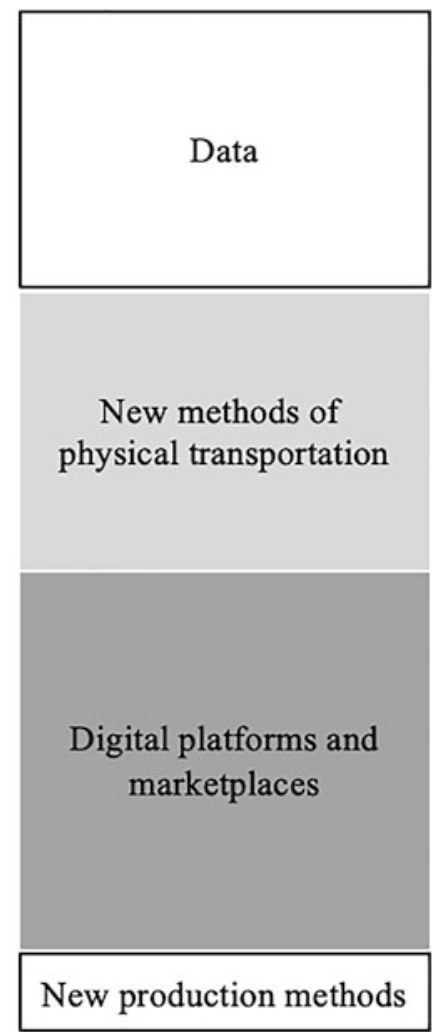

Digital technological innovation

Data collection and treatment

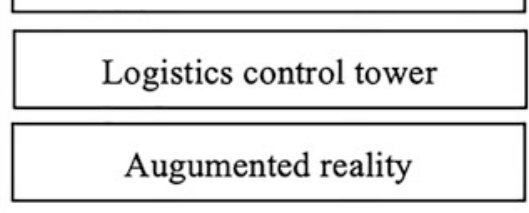

Driverless transport systems.

Robots

\section{Drones}

Big cross-border platform

Shared transport capacity

Shared warehouse capacity

Crowdsourcing

3D printing

Fig. 1 Logistics 4.0 building blocks (Skapinyecz et al. 2018)

demonstrate how they influence human decision-making. After examining the effects of cognitive biases, we derive initial propositions for further research in this field.

\section{Literature Review}

\subsection{The Human Decision-Making Process and Cognitive Biases}

The human decision-making process has been the focus of research in various fields, such as psychology and strategic and behavioral organization management. A 
Table 1 Relevant cognitive biases

\begin{tabular}{l|l}
\hline Category of cognitive bias & Description \\
\hline Memory bias & $\begin{array}{l}\text { Group of cognitive biases related to information storage and } \\
\text { availability }\end{array}$ \\
\hline Statistical bias & $\begin{array}{l}\text { Tendency to overestimate or underestimate certain statistical } \\
\text { parameters }\end{array}$ \\
\hline Adjustment bias & $\begin{array}{l}\text { Tendency to stick to the first information available or to a } \\
\text { reference point }\end{array}$ \\
\hline Presentation bias & Decision based on the display of information \\
\hline Situational bias & The way a person responds to a general situation \\
\hline
\end{tabular}

decision is a situational response that consists of three parts (Tversky and Kahneman 1974a):

1. More than one possible choice of action is under consideration in the choice set.

2. The decision maker can form expectations of future events and outcomes ensuing from each course of action, which are describable by degrees of belief or probabilities.

3. The consequences of the possible outcomes are assessable on an evaluative continuum determined by current goals and personal values.

The broad scope and various linked variables can make decisions in production logistics very complex. Most decisions have to be made to optimize business activities and, in some cases, only have an impact after a prolonged period. Tversky and Kahneman (1974b) demonstrated that humans make systematic errors in particular decision environments and introduced the term cognitive bias to denote subconscious errors in human decision-making. Reflecting on cognitive biases and their impact is essential during the decision-making process. Individuals make irrational decisions based on their respective backgrounds, knowledge, experiences, and attitudes (Bendul and Zahner 2019; Arlinghaus et al. 2020). We mapped several cognitive biases from each category of the most important main groups of Arnott's (MacCarthy and Fernandes 2000) categorization, some of which examined in more detail in this study, to the three decision types in production logistics. Table 1 shows the categorization of cognitive biases effects by Arnott (MacCarthy and Fernandes 2000) and in which decision-making area they have an impact.

\subsection{Decisions in Production Logistics}

Production logistics describes planning, coordination, transportation, and similar activities related to manufacturing. Production logistics does not function in any set way since it is contingent on each company's manufacturing environment and products (Jonsson and Mattson 2003; Fleischmann et al. 2005). This concurs with 


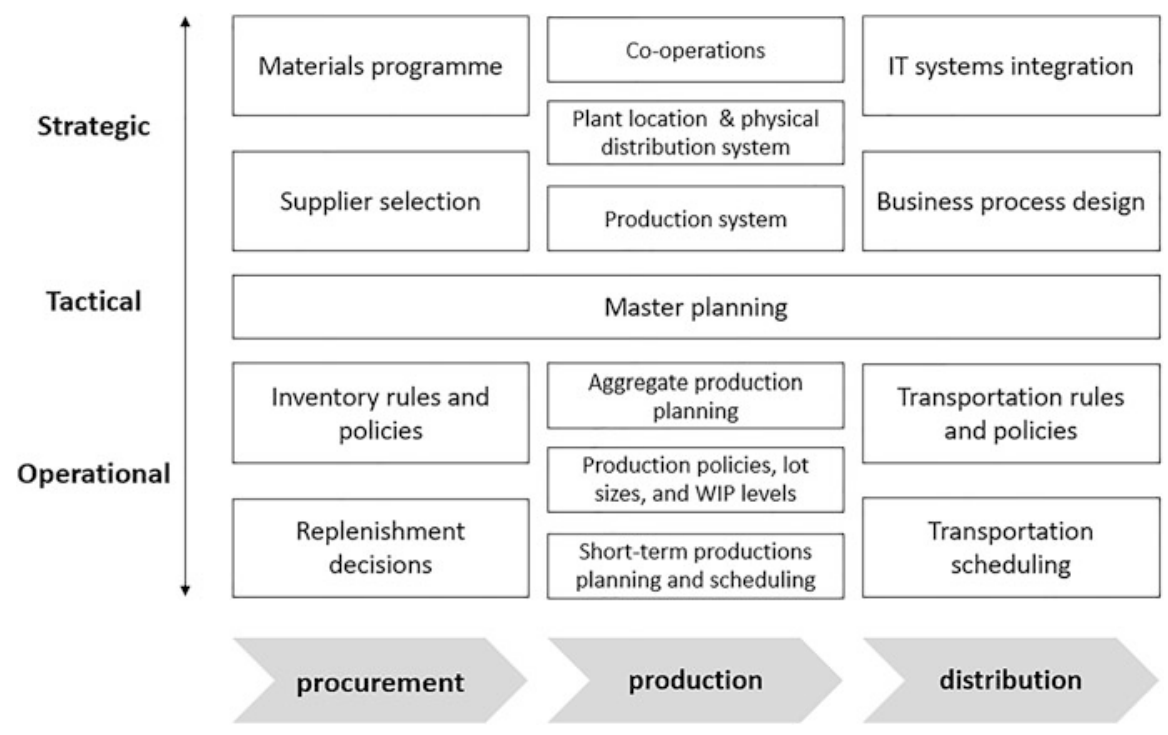

Fig. 2 Production logistics decision areas (based on Fleischmann et al. (Arnott 2006))

Semini et al. (2006) who point out that "manufacturing logistics encompasses aspects of several overlapping fields, including operations and production management, logistics and supply chain management, and advanced planning." This field's diversity has spawned an abundance of different approaches. Some authors, such as Chan (2005), view production planning and control as the crux of production logistics. Others, such as Strandhagen et al. (2006), employ a modified version of the supply chain planning matrix developed by Fleischmann et al. (Arnott 2006).

Figure 2 presents the range of production logistics that require human decisionmaking. Decision types can be categorized as strategic, tactical, and operational based on the decision horizon. Strategic decisions typically have a long-term horizon, operational decisions a short-term horizon, and tactical decisions a mediumterm horizon.

We added the decision horizon to the supply chain planning matrix of Fleischmann et al. (Arnott 2006) and mapped the different decision types to the typical tasks in production logistics as illustrated in Fig. 2.

Production logistics decisions are classifiable by the decision horizon introduced (Ghiani et al. 2004). Decisions are made iteratively and hierarchically from the strategic to the tactical and the operational level. These decision-making levels are identified and explained with examples in Table 2 (Seifi 2011). 


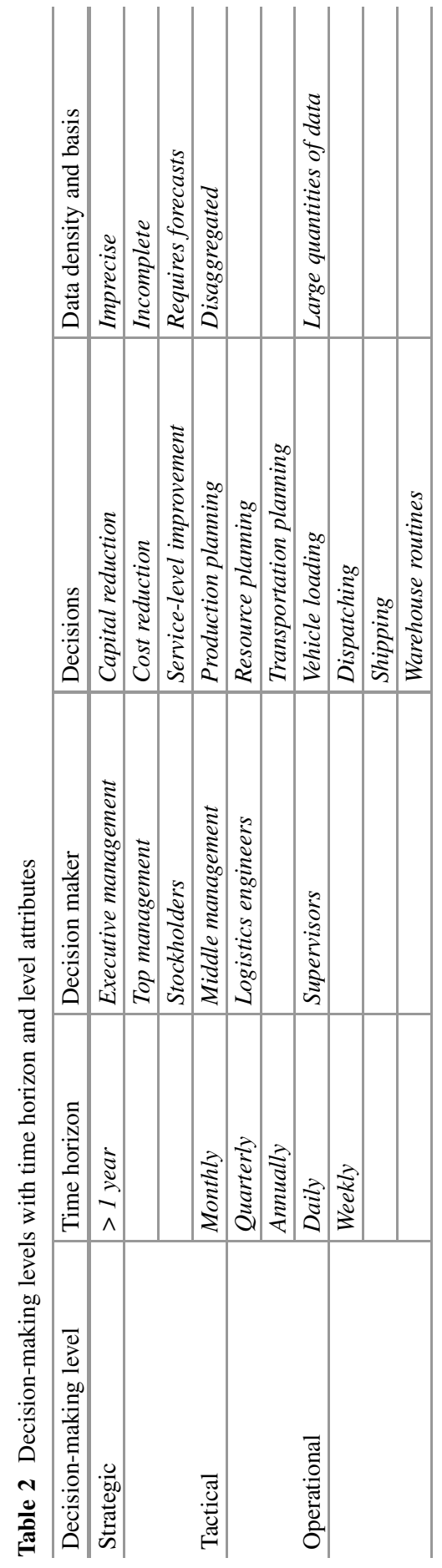




\section{Conceptual Framework of Distorted Human Decision-Making in Production Logistics}

\subsection{Strategic Decisions}

Memory Biases: Imaginability Bias Imaginability bias describes individuals' assumption that events they can easily imagine are more probable. Individuals' own attitudes and imagination thus influence the assessment of the probability of a risk occurrence (Taylor and Thompson 1982), A company is more likely to install an integrated IT system to track spare parts across units if the decision maker is IT savvy than if the decision maker is incapable of imagining such a system or any added value from its implementation.

Adjustment Biases: Conservatism Bias Individuals that weight new information less than initial information are displaying conservatism bias (Pompian 2012). In the case of strategic decision this often occurs that persons stick to already known and common solutions and avoid completely new methods. For instance, they might pay less attention to new production logistics processes such as additive manufacturing despite all its advantages.

Confidence Biases: Confirmation Bias The reflection of a decision maker's attitude in a decision stems from confirmation bias. Arguments that support personal opinion are weighted more heavily than others that do not (Wheeler and Arunachalam 2008). For instance, buyers might select vendors based on their personal preferences (Table 3).

Since some of the many decisions made at the strategic level are fraught with uncertainty, they depend on the decision maker's skills and background. We therefore propose:

P1: Cognitive biases related to the decision maker's skills and background heavily influence strategic decisions based on uncertain data.

Table 3 Cognitive biases in strategic decisions

\begin{tabular}{l|l}
\hline \multicolumn{1}{l|}{ Cognitive biases } & Main decision(s) at the strategic level \\
\hline Memory bias & Which relevant facts deserve further consideration? \\
\hline \multicolumn{1}{l}{ Imaginability bias } & Am I the best person to judge? \\
\hline \begin{tabular}{l} 
Adjustment bias \\
\hline$\bullet$ Conservatism bias
\end{tabular} & What changed since the last period under review? \\
\hline Confidence bias & Are there other targeted options? \\
\hline
\end{tabular}




\subsection{Tactical Decisions}

Presentation Biases: Ambiguity Effect The ambiguity effect describes humans' tendency to favor the seemingly simplest option over more complex options (Ellsberg 1961). Practitioners prefer quick and easy solutions that can be found and applied quickly. Exemplarily, the lead time syndrome shows this connection. When facing decreasing due date reliability, the most simple looking option is to adapt planned lead times. Other influencing effects are consequently underestimated.

Confidence Biases: Illusion of Control The illusion of control describes individuals' tendency to overestimate their ability to solve difficult problems (Brenner et al. 1996). People tend to overestimate their own abilities as well as their own plans. This is especially true when production and logistics parameters are monitored insufficiently because the decision maker deems them unimportant.

Situational Biases: Ostrich Effect The ostrich effect describes people's habit of ignoring obviously negative information in order to advance certain (e.g., their own) interests (Karlsson et al. 2009). For instance, the workload of a machine that is heavily utilized but also prone to malfunction is not reduced and the risk of jeopardizing due date reliability is ignored (Table 4).

Although the base data can be used for decisions, the decision maker's (personal) skills also influence decision depending on its scope. We therefore propose:

P2: Both cognitive biases that are related to personality factors and influence the treatment of data influence medium-term decisions.

\subsection{Operational Decisions}

Adjustment Biases: Anchoring Effect This cognitive bias describes the treatment of initial information as an "anchor" to which individuals hold fast (Tversky and Kahnemann 1974). This might result in new technologies, such as automated guided vehicles, not being considered in transportation planning, for instance.

Table 4 Cognitive biases in tactical decisions

\begin{tabular}{l|l}
\hline & Main decision(s) at the tactical level \\
\hline Cognitive biases & Which relevant facts deserve further consideration? \\
\hline Presentation bias & \multicolumn{2}{|l}{ Ambiguity effect } & Are there other options that will create future added value? \\
\hline Confidence bias & Is the project, schedule or the like still realistic? \\
\hline \multicolumn{1}{|l}{ Illusion of control } & Is all information assessed equally? \\
\hline Situational bias & Ostrich effect
\end{tabular}


Table 5 Cognitive biases in operational decisions

\begin{tabular}{l|l}
\hline \multicolumn{1}{|l}{} & Main decision(s) at the operational level \\
\hline Cognitive biases & Which relevant facts deserve further consideration? \\
\hline Adjustment bias & What changed during a period of time? \\
\hline \multicolumn{1}{|l}{ Anchoring effect } & Has the correct context been factored in everywhere? \\
\hline Statistical bias \\
\hline$\bullet$ Correlation bias & Is there useless data? \\
\hline Situational bias & Complexity effect
\end{tabular}

Statistical Biases: Correlation Bias Humans assessing two concurrent risks tend to overestimate their probability of occurrence when they have occurred previously (Kahneman 2002). This is called correlation bias and makes it difficult to identify triggers since a correlation of both risks is assumed. Process difficulties or improvements (Event 1) are thus associated with the new solution that has just been implemented (Event 2). Since this generates false assumptions about correlation, it can result in incorrect assumptions, especially in the case of planning updated daily because individuals might believe that one parameter indicates good schedule adherence for a product, even though it has no real correlation to schedule adherence.

Situational Biases: Complexity Effect The complexity effect describes individuals' bias when they are under time pressure or overloaded by information (Ordonez and Benson 1997). Their assessment of various factors' effects and potential impact is consequently unduly complex since they often link various parameters. This can result in incorrect decisions, especially in conjunction with time pressure, e.g., in replenishment because of shortages or unduly high inventory levels, and thus in high costs for storage space or problems caused by missing material (Table 5).

Since the decision maker can access a large quantity of data at the operational level, we propose that:

P3: As data density increases, these cognitive biases affect decisions about uncertainty, data handling, and time pressure adversely.

\section{Conclusion}

We identified potential decisions in production logistics and correlated them with potential cognitive biases in order to answer to the research question introduced: Which types of decisions in production logistics are influenced by cognitive biases and how do these biases affect production logistics performance? After identifying a correlation between the decision-making level and the possible type of bias, we advanced three propositions that provide initial insights on potential correlations in 
decision-making in production logistics. A closer examination of the different levels of production logistics reveals that the main groups of cognitive biases cannot be assigned to specific levels in the decision-making process. Categorizing cognitive biases based on the density of data for the potential decision would constitute a better approach since some effects stem more from data handling and others more from the decision maker's personality.

Since biases correlate with multiple levels of logistics decisions, we see a need for further research in this area. Experiments with practitioners would help assign cognitive biases to levels and decisions better and enable drawing conclusions about the extent to which personal or individual factors influence decisions when all subjects have similar backgrounds and perform identical tasks.

\section{References}

Arlinghaus, J.C., et al.: The influence of cognitive biases on supply chain risk management in the context of digitalization projects. In: Freitag, M., Haasis, H.D., Kotzab, H., Pannek, J. (eds.) Dynamics in Logistics. LDIC 2020. Lecture Notes in Logistics, pp. 137-147. Springer, Cham (2020)

Arnott, D.: Cognitive biases and decision support systems development: a design science approach. Inf. Syst. J. 16(1), 55-78 (2006)

Bamberger, V., et al.: Logistics 4.0 - facing digitalization - driven disruption. PRism. 1, 38-51 (2017)

Bazerman, M.H., et al.: Cognitions and behavior in asymmetric social dilemmas: a comparison of two cultures. J. Appl. Psychol. 87(1), 87-95 (2002)

Bendul, J., Knollmann, M.: The human factor in production planning and control: considering human needs in computer aided decision support systems. Int. J. Manuf. Technol. Manag. 30(5), 346-368 (2016)

Bendul, J.C., Zahner, J.H.: Understanding the influence of cognitive biases in production panning and control. In: Ahram, T.Z., et al. (eds.) Human Systems Engineering and Design II. IHSED 2019. Advances in Intelligent Systems and Computing, vol. 1026, pp. 280-285. Springer, Cham (2019)

Brenner, L.A., et al.: On the evaluation of one-sided evidence. J. Behav. Decis. Mak. 9(1), 59-70 (1996)

Brettel, M., et al.: Fostering creativity in new product development through entrepreneurial decision making. Creat. Innov. Manag. 23(4), 351-511 (2014)

Chan, J.W.K.: Competitive strategies and manufacturing logistics - an empirical study of Hong Kong manufacturers. Int. J. Phys. Distrib. Logist. Manag. 35(1), 20-43 (2005)

CSCMP Supply Chain Management Definition and Glossary. https://cscmp.org/CSCMP/Educate/ SCM_Definitions_and_Glossary_of_Terms.aspx. Last accessed 06 Aug 2021

De Felice, F., Petrillo. A.: Green policy in a manufacturing system. In: IEEE (eds.) 2nd International Conference on Communications, Computing and Control Applications, CCCA, pp. 1-6 (2012)

Ellsberg, D.: Risk, ambiguity, and the savage axioms. Q. J. Econ. 75(4), 643-669 (1961)

Erol, S., et al.: Tangible industry 4.0: a scenario-based approach to learning for the future of production. Procedia CIRP. 54, 13-18 (2016) 6th CIRP Conference on Learning Factories

Fleischmann, B., et al.: Supply Chain Management and Advanced Planning, 3rd edn. Springer, Berlin (2005)

Ghiani, G., et al.: Introduction to Logistic Systems Planning and Control. Wiley, West Sussex (2004) 
Johnson, D.F., et al.: Contemporary Logistics. Prentice Hall, New Jersey (1999)

Jonsson, P., Mattson, S.A.: The implications of fit between planning environments and manufacturing planning and control methods. Int. J. Oper. Prod. Manag. 23(8), 872-900 (2003)

Kahneman, D.: A perspective on intuitive judgment and choice: mapping bounded rationality. Novel price literature. Am. Psychol. 58(9), 697-721 (2002)

Karlsson, N., et al.: The ostrich effect: selective attention to information. J. Risk Uncertain. 38, 95-115 (2009)

MacCarthy, B.L., Fernandes, F.C.F.: A multi-dimensional classification of production systems for the design and selection of production planning and control systems. Prod. Plan. Control. 11(5), 481-496 (2000)

Ordonez, L., Benson, L.: Decisions under time pressure: how time constraint affects risky decision making. Organ. Behav. Hum. Decis. Process. 71(2), 121-140 (1997)

Pompian, M.M.: Conservatism bias. In: Behavioral Finance and Wealth Management: how to Build Investment Strategies that Account for Investor Biases, 2nd edn, pp. 63-71. Wiley, Hoboken, NJ (2012)

Rushton, A., et al.: The Handbook of Logistics and Distribution Management. Kogan Page, London (2006)

Seifi, M.S.: Logistics strategic decisions. In: Logistics Operations and Management, Concepts and Models, pp. 43-53. Elsevier, Amsterdam (2011)

Semini, M., et al.: Applications of discrete-event simulation to support manufacturing logistics decision-making: a survey. In: Perrone, L.F., et al. (eds.) Proceedings of the 2006 Winter Simulation Conference, pp. 1946-1953. WSC, Monterey, CA (2006)

Skapinyecz, R., et al.: Logistic aspects of industry 4.0. IOP Conf. Ser. Mater. Sci. Eng. 448, 012014 (2018)

Stanovich, K.E., West, R.E.: Individual differences in rational thought. J. Exp. Psychol. Gen. 127(2), 161-188 (1998)

Strandhagen, J. O. et al.: Which manufacturing logistics decisions are supported by operational research? A literature survey. IFIP International Federation for Information Processing (2006)

Taylor, S.E., Thompson, S.C.: Stalking the elusive "vividness" effect. Psychol. Rev. 89(2), 155181 (1982)

Tversky, A., Kahneman, D.: Judgment under uncertainty: heuristics and biases. Science. 18(4157), 1124-1131 (1974a)

Tversky, A., Kahneman, D.: Judgment under uncertainty: heuristics and biases. Science. 18(4157), 1112-1131 (1974b)

Tversky, A., Kahnemann, D.: Judgement under uncertainty: heuristics and biases. Science. 185415, 1124-1131 (1974)

Wheeler, P.R., Arunachalam, V.: The effects of decision aid design on the information search strategies and confirmation bias of tax professionals. Behav. Res. Account. 20(1), 131-145 (2008)

Open Access This chapter is licensed under the terms of the Creative Commons Attribution 4.0 International License (http://creativecommons.org/licenses/by/4.0/), which permits use, sharing, adaptation, distribution and reproduction in any medium or format, as long as you give appropriate credit to the original author(s) and the source, provide a link to the Creative Commons license and indicate if changes were made.

The images or other third party material in this chapter are included in the chapter's Creative Commons license, unless indicated otherwise in a credit line to the material. If material is not included in the chapter's Creative Commons license and your intended use is not permitted by statutory regulation or exceeds the permitted use, you will need to obtain permission directly from the copyright holder. 